\title{
BMJ Open Trainee perspective of the causes of stress and burnout in surgical training: a qualitative study from Wales
}

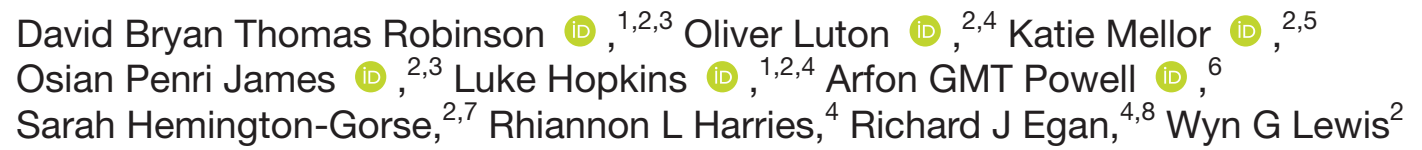

To cite: Robinson DBT, Luton 0, Mellor K, et al. Trainee perspective of the causes of stress and burnout in surgical training: a qualitative study from Wales. BMJ Open 2021;11:e045150. doi:10.1136/ bmjopen-2020-045150

- Prepublication history and additional online supplemental material for this paper are available online. To view these files, please visit the journal online. (http://dx.doi.org/10. 1136/bmjopen-2020-045150)

Received 24 September 2020 Accepted 17 June 2021

Check for updates

(c) Author(s) (or their employer(s)) 2021. Re-use permitted under CC BY-NC. No commercial re-use. See rights and permissions. Published by BMJ.

${ }^{1}$ Postgraduate Research Department, Cardiff University School of Medicine, Cardiff, UK ${ }^{2}$ School of Surgery, NHS Wales Health Education and Improvement Wales, Nantgarw UK

${ }^{3}$ General Surgery, Royal Gwent Hospital, Newport, UK ${ }^{4}$ General Surgery, Morriston Hospital, Swansea, UK

${ }^{5}$ General Surgery, Ysbyty

Gwynedd, Bangor, UK

${ }^{6}$ Division of Cancer and Genetics, Cardiff University School of Medicine, Cardiff, UK ${ }^{7}$ Department of Plastic Surgery, Morriston Hospital, Swansea, UK ${ }^{8}$ Swansea University College of Medicine, Swansea, UK

Correspondence to

Dr Arfon GMT Powell;

powella16@cardiff.ac.uk

\section{ABSTRACT}

Objectives Stress and burn-out among surgical trainees has been reported most prevalent in core surgical trainees (CST) and female trainees in particular. This study aimed to identify factors perceived by CSTs to be associated with stress and burnout in those at risk.

Design An open-ended questionnaire was distributed to 79 CSTs and two researchers categorised responses independently, according to Michie's model of workplace stress.

Setting A UK regional postgraduate medical region (Wales).

Participants Sixty-three responses were received; 42 males, 21 females. The response rate was $79.7 \%$. Results Inter-rater reliability was good $(\mathrm{k}=0.792$ $(79.2 \%), p<0.001)$. The most common theme of Michie's model related to CST stress and burnout was career development, with most statements associated with curriculum, examination and academic demands required to attain a CST certificate of completion of training, and higher surgical national training number appointment. This was closely followed by those intrinsic to the job with recurrent discussion around the difficulties balancing work perceived to be service provision (ward work and on-calls), outpatient clinic and operative experience. Conversely, the most common themes relevant to stress and burnout among female trainees were associated with relationships at work (primarily the male-dominated nature of surgery), extraorganisational factors (family-work life balance) and individual characteristics (personality and physiological differences).

Conclusion CSTs' perceptions regarding the causes of National Health Service related stress and burnout are numerous, and these findings provide a basis for the development of targeted stressor counter-measures to improve training and well-being.

\section{INTRODUCTION}

The relationship between workplace stress and occupational burn-out is a complex paradigm. Stress is both important in overcoming certain challenges yet damaging in excess quantity. ${ }^{1}$ Prolonged exposure to excessive stressors can result in individuals developing occupational burnout, characterised by emotional exhaustion, depersonalisation and
Strengths and limitations of this study

- This study uses an established workplace model for stress which has previously been used effectively in similar cohorts (FY1 doctors).

- The response rate of the cohort studied is good and the inter-rater reliability of the statements provided ranged from good to excellent.

- It provides direct trainee perspectives of the causes of stress and builds on previous work identifying its high prevalence, filling a gap in the literature pertaining to this cohort.

- Questionnaire methodology has inherent disadvantages, primarily that two-way communication cannot be achieved, to clarify the meaning underlying a respondent's answer and vice-versa, respondents may struggle to gain clarity regarding the focus of questions asked.

- Participants were from a single UK regional postgraduate medical region (Wales).

a diminished sense of personal accomplishment. $^{2}$ The WHO's 10th revision of the International Classification of Diseases (ICD-10) added burn-out as a recognised occupational phenomenon, with a more detailed definition in the most recent iteration (ICD-11). According to WHO, 'burn-out is a syndrome conceptualised as resulting from chronic workplace stress that has not been successfully managed'. 3 Burn-out among surgeons has been identified as a growing issue with serious manifestations including effects on mental health (depression and anxiety), relationships, the potential for alcohol/substance addiction and even suicide. ${ }^{4}$

Michie proposed a conceptual model to categorise the causes of stress at work into themes based on the content and context of stressors, as originally outlined by Murphy. ${ }^{56}$ Michie developed Murphy's work by examining the relationship between the themes and their impact on individuals, manifesting as symptoms of occupational ill health, before 


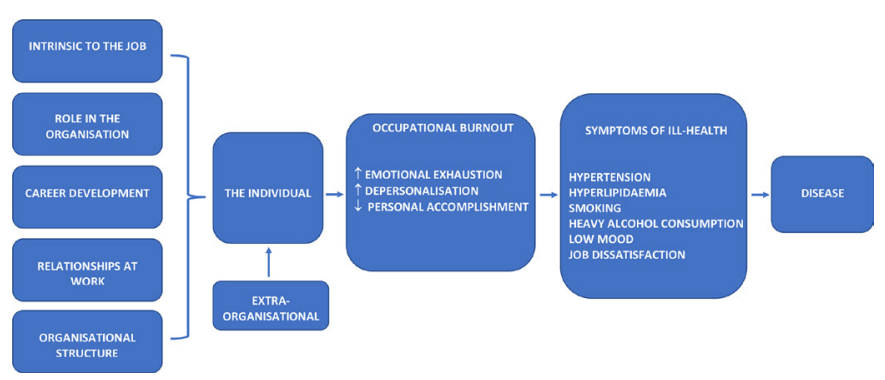

Figure 1 Relationship between workplace sources of stress, the individual and subsequent disease as defined by Michie adapted to illustrate the relationship between stress and burnout in the sequence of events. ${ }^{4}$

presenting as defined diseases such as mental or cardiovascular disease. ${ }^{5-7}$ Figure 1 illustrates the relationship between workplace sources of stress, the individual and subsequent disease as defined by Michie but has been adapted to highlight the relationship of occupational burnout within the sequence of events. Tallentire et al developed this thesis to explore the stressors applicable to postgraduate year 1 (PGY1) doctors, and reported that Michie's model of workplace stress was effective in analysing the stressors relevant to PGY1 doctors, identifying uncertainty as a recurring issue, and common to all themes defined by the model.

It was previously reported in 2020 that burn-out among Wales' surgical trainees was common, with $59 \%$ of respondents meeting the criteria for high burn-out in at least one domain. Moreover, multivariable analysis revealed that high burn-out in all three domains was associated with core surgical trainee (CST) grade and female gender. ${ }^{8}$ This study aimed to identify the potential stressors that contribute to the higher levels of occupational burn-out perceived by CSTs using Michie's model as a framework.

\section{MATERIAL AND METHODS}

An open-ended questionnaire was distributed to all CSTs $(\mathrm{n}=79)$ enrolled in the training programme at Health Education and Improvement Wales' 2019 Annual Review of Competency Progression (ARCP). The questionnaires were completed voluntarily and anonymously in a holding room prior to the trainees' ARCP. Only 2-3 individuals were present in the holding room at any given time which reduced the possibility of intertrainee communication while completing the questionnaire. The questionnaire outlined two questions in line with the stress and burn-out findings from the aforementioned study investigating stress and burnout among surgical trainees in Wales. First: why did CSTs consider that their specific grade was associated with higher levels of burnout? Second: why did CSTs consider that female gender was associated with higher levels of burn-out? A further option allowed for other comments, and these statements were categorised into one of the main domains pertaining to either CST or female trainee risk of stress and burn-out.

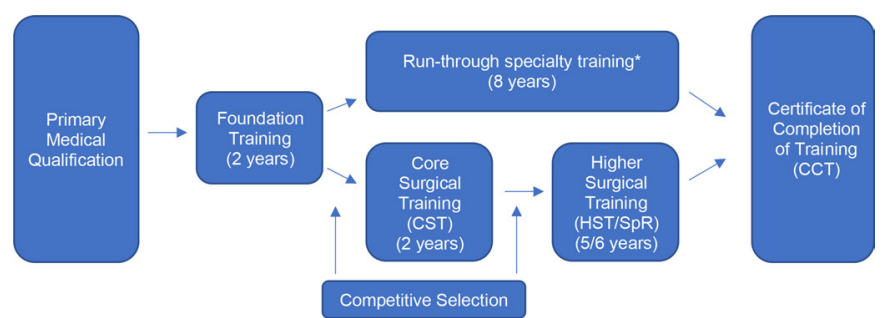

Figure 2 Surgical training progression pathway in the UK. *Select surgical specialties only-The majority of surgical training programmes go through a 2-year period of core surgical training, followed by a 5/6-year higher surgical training programme culminating in certification of completion of training.

Two analyses were performed; one related to the results of each question. Data collected was transcribed and classified into themes using an adapted version of Michie's model of workplace stress as described by Tallentire $e t a l$ with the additional caveat that uncertainty was a recurrent and common PGY1 doctor's theme, and was therefore added as an independent category. ${ }^{57}$ Figure 2 demonstrates the standard surgical training progression pathway in the UK.

\section{Conceptual framework}

This study builds on the existing literature regarding occupational stress and burn-out in the medical arena where information relating to UK surgical trainees is sparse. Recently, it has been reported that CST and female trainees in particular were identified as being the most at-risk of occupational burn-out. ${ }^{8}$ While causality may be inferred, the approach employed in this study provides a first-hand account of perceived causes of stress, that accumulate resulting in burn-out among susceptible individuals using the conceptional typology described by Michie.

\section{Statistical analysis}

To ensure accurate reporting during the categorisation of the causes of stress and burnout described by the CSTs, two researchers (DBTR and OL) independently analysed and categorised the statements. Agreement between researchers was analysed using the kappa statistic $(\mathrm{k}){ }^{9}$ Altman's rating of strength of agreement was used: $k<0.20$ (poor), $\mathrm{k}=0.21-0.40$ (fair), $\mathrm{k}=0.41-0.60 \quad$ (moderate), $0.61-0.80$ (good) and $0.81-1.00$ (excellent). ${ }^{10}$ When there was disagreement regarding statements, consensus was achieved by discussion between the two researchers. Data were collected and analysed in SPSS V.25 (SPSS, IBM).

\section{Patient and public involvement}

All participants of this study were CSTs. There was no patient or public involvement.

\section{RESULTS}

A total of 63 responses were received (response rate $79.7 \%$ ); of which all respondents (42 male, 21 female) 
answered the question related to higher burnout in CSTs, whereas 37 trainees (19 male, 18 female) answered the question regarding potential causes of higher burn-out among female trainees. Where reasons for non-completion were given by males, it was because they considered that they could not comment on behalf of their female peers. The number of statements generated related to causes of stress and burnout among CSTs and females totalled 192 and 71, respectively. In addition, five individuals (four female, one male) commented that they felt there were no differences related to gender and that the issues faced were the same regardless.

Overall statement agreement (217/263, k=0.792 $(79.2 \%), \quad \mathrm{p}<0.001)$ and agreement regarding CST burn-out aetiology $(155 / 192, \mathrm{k}=0.759(75.9 \%), \mathrm{p}<0.001)$ between researchers was good, with agreement regarding female trainees rated as excellent $(62 / 71, \quad \mathrm{k}=0.821$ $(82.1 \%), \mathrm{p}<0.001) .{ }^{10}$

Online supplemental appendix A and B outline all statements transcribed from the trainee questionnaires classified by the relevant themes of Michie's model for causes of burnout related to both CSTs and females, respectively. Responses have been divided related to respondent gender. No statements relating to stress and burn-out in females were received for the following themes: intrinsic to the job, career development, and uncertainty.

\section{Intrinsic to the job}

This cohort of CSTs made several statements related to their day-to-day workload with particular reference to the balance between meeting all of their clinical duties and fulfilling training requirements. Representative quotes include;

Trying to maintain surgical training requirements whilst ensuring that the service aspect of the job is not neglected.

The demand for WBAs (workplace-based assessments), operating numbers etc. is much higher than for SpRs (specialist registrars), but we have more responsibilities in terms of ward duties therefore have less opportunity to do this.

Numerous clinical duties in the form of ward rounds, inpatient care while simultaneously balancing clinics and theatre.

Necessary to come in on days off in order to attend theatre lists because it is not possible to get off the wards during the normal working hours.

\section{Role in the organisation}

Trainees repeatedly commented on their intermediate position; they are neither the most junior nor the most senior members of the team and this often results in a greater workload compared with other team members.

Strange dynamic as CST required to step down (act as foundation level) and step up (act as $\mathrm{SpR}$ ) as per unit dynamics.
Intermediate hospital position therefore have to balance theatres, clinics and wards.

They also frequently commented on the increased expectations compared with their time as foundation doctors.

The step up between F2 and CST is significant with regards to what you are supposed to achieve.

More is expected of you from day 1 of each new job, regardless of previous experience.

Tangible step up in terms of responsibilities, duties and requirements.

Four statements were made related to this theme with regard to female burn-out. All four relate to a perception of greater demands/expectations on female trainees.

Females have to work harder to prove themselves as trustworthy or competent.

As we have fewer female trainees I think there are greater expectations on female colleagues.

\section{Career development}

This theme contained the most statements related to the sources of stress in CSTs. Common factors related to the heavy additional workload associated with this training period in terms of professional examinations and portfolio development.

Balancing examinations, WBAs and developing operative skills is stressful and time consuming.

Compared with peers in medical specialities there is a much higher number of mandatory assessments including WBAs, research and procedures.

The additional burden of extracurricular activities and maintaining academic work can result in greater stress and mental strain.

Other comments also touched on the financial burden of preparing for higher surgical national training number (NTN) applications.

Courses are expensive and the study budget just about covers boot camp but CSTs need to attend multiple workshops and conferences which adds to the sudden increase in financial burden.

Higher cost of compulsory exams/courses compared with other specialities.

\section{Relationships at work}

Trainees' statements regarding relationships at work were primarily related to a lack of senior/consultant support or engagement.

When approaching seniors regarding the lack of training opportunities, I was often faced with remarks such as 'be more proactive', 'utilise your leave' or even 'just bear with it and soon it will be over when you are a SpR'. 
Difficulties engaging with some seniors to complete WBAs.

Lack of senior support on call.

This theme received the most statements with regard to the perceived gender related causes of burn-out, with many commenting on the male dominated nature of surgical departments as a significant source of female stress and burn-out.

Very male dominated departments can be intimidating for female trainees and discouraging.

Fewer female higher trainees/consultants to provide support.

Relationships with patients, nurses and fellow doctors have also been cited.

Patients often confuse female doctors for nurses even when out of scrubs and with a stethoscope around their neck. When going around (the ward) with a F1/ F2 who is male, they (the patient) assume they are more senior and engage/receive them better......

Nurses are more receptive to male doctors than female.

Males form informal and friendly relationships easier with consultants (mostly male).

\section{Organisational structure and climate}

Staffing issues have been repeatedly mentioned as a source of CST stress and burnout. When combined with the demands of the curriculum, rota gaps and staff shortages, this can often lead to trainees needing to do overtime, frequently unpaid.

Staff shortages, therefore often used for service provision.

Rota gaps leading to a lack of training opportunities.

Have to work late or in own time to ensure we achieve the minimum requirements.

An overlap between this theme and relationships at work was noted with regard to perceived gender related issues. Similarly, it was felt that the organisational climate played a role in the fundamental reasoning that departments were male orientated/dominated.

Male orientate/dominated environment.

Fewer female examples of senior leadership relative to male.

\section{The individual}

This was an uncommon theme with regard to general causes of CST stress and burnout with only four statements fitting into this category. They were related to what was perceived to be the characteristics of a surgeon.

Natural perfectionism amongst types of people who choose surgery.

Doctors are poor at asking for help when they need it.
Conversely, it was the third most common theme related to contributory factors in the development of female related stress and burnout, and related to the more diligent/perfectionist nature of females compared with males.

Harder to get surgical opportunities as more diligent with ward work.

Females find it harder getting away from the wards if it means burdening others.

Greater degree of perfectionism amongst female CSTs.

\section{Extraorganisational}

A recurrent theme pertinent to both CST and female trainee burn-out were those surrounding work-life/family balance.

With increasing age and life progression it is harder to maintain a work-life balance.

Balancing family life and preparing a competitive portfolio can be challenging.

Stress related to planning on starting a family and the additional complexities of LTFT (Less Than Full Time) training and maternity leave.

Moreover, in addition to the usual contracted hours of work, it was considered that personal/free time was further limited by extracurricular necessities overlapping with those associated with career progression.

During times of relaxation I feel the need to work on portfolios/projects which means 'down time' is reduced.

Expectations to fulfil both educational and clinical responsibilities whilst being able to take 'down' time.

\section{Uncertainty}

Uncertainty was not a major stressor reported by CSTs $(n=4)$ and did not align with any statements related to gender. CST statements mapping to uncertainty were all related to future career development and progression to higher surgical training.

Uncertainty about future progression-either fall off/choose other specialities due to pressure or succeed therefore I feel that specialist training posts select the swimmers.

Uncertainty over location/availability of registrar jobs.

\section{DISCUSSION}

This is the first study to report trainee perceptions regarding the potential causes of stress and burnout among UK CSTs, with an added focus on the additional challenges faced by women. All the themes of Michie's model were populated by the respondent's answers, with the most common emerging theme being career 
development, closely followed by factors intrinsic to the day-to-day job of a junior surgeon. The largest contributor to CST stress and burn-out was perceived to be the large size of the workload, both in the clinical setting, and that required to build a competitive Curriculum Vitae profile in preparation for NTN selection. Multiple themes were not populated in the course of female trainee analysis, arguably because the majority of stressors faced by women are largely similar to those relevant to all surgical trainees. However, given that women were previously reported to be at greater risk of burn-out, it was considered important to identify issues that may drive this gender-related discrepancy. ${ }^{8}$ The additional challenges faced by women identified in this study would appear to be connected with a working environment that is heavily male dominated, and the consequences that may therefore ensue, including lack of senior support and role models, and male gender bias. Moreover, managing home life and family planning, in tandem with surgical career progression demands, was deemed to be a significant factor which was more pertinent to female trainees.

Tallentire et al reported that career uncertainty was a common factor related to all themes among PGY1 doctors, an understandable finding, given that it has been suggested that a doctor's first year after qualification is the most stressful time of their careers. ${ }^{711}$ However, this study suggests that by the time of CST, this underlying uncertainty has largely disappeared; CSTs know what they want-the challenge is to deliver it.

In 2019, the Association of Surgeons in Training published the findings of their questionnaire survey exploring the factors that attracted medical students and doctors into surgical training. As well as the positive factors, they outlined potential barriers and areas ripe for improvement, in order to make surgical careers more attractive. It was recommended that a greater emphasis on training during working hours, less financial burden, and more focus on work-life balance, should make surgery a more appealing career choice. Moreover, it was reported that whereas male respondents stated that a greater sense of belonging, condensed training programmes, and increased opportunity for surgical rotations earlier in their careers, would improve recruitment and retention, females stated that greater emphasis on work-life balance would be more effective. ${ }^{12}$ These observations align with the findings of this study and further support the need for existential flexibility during CST.

In 2017, the General Medical Council reported that surgery is presently the most male-dominated of all medical specialties. Only one in eight surgeons on the specialist register is female, and despite comprising $55 \%$ of medical students, and $58 \%$ of doctors in UK training, females only account for one in three surgeons in training. ${ }^{13}$ Although this represents an increase from one in four trainees in 2012, it clearly remains an issue. Even if the number of female surgical trainees continues to increase at the current trajectory, given the time taken to reach consultant status, the male numbered dominated workplace, will likely endure for some time yet.

A diverse workforce has been shown in the business sector to result in improved productivity, innovation, decision making, employee retention and satisfaction, with gender diverse companies outperforming their less diverse competitors. ${ }^{14}$ It has been suggested that the benefits observed in other industries may translate to better team clinical performance, with different but equally important skills, along with an alternative perspective, resulting in better and safer patient care. ${ }^{15}$ Wallis et al reported a comparison of the postoperative outcomes related to male and female surgeons; no significant differences in operative complications or readmission rates were observed, but patients treated by female surgeons were less likely to suffer operative mortality and die within 30 days of surgery (OR 0.88, $\mathrm{p}=0.04) .{ }^{16}$ Any reasonable observer would surely agree that the modern surgical unit has to find ways of counteracting the negative attributes associated with a male heavy department, and ensure that all teams welcome and support women, so that equity for all is ensured and the benefits of a gender diverse team are realised.

This paper highlights the factors precipitating the high levels of burnout among junior surgical trainees, but using the aetiology outlined further work is required to develop solutions aimed at this multifactorial issue. One possible intervention has already started, with the introduction of the Improving Surgical Training (IST) pilot programme. This 2-year programme (a replacement for CST) offers themed run-through training, with guaranteed progression from CST to Higher Surgical Training, if trainees are deemed to have attained all appropriate competencies by their CST2 year ARCP. This may help combat the biggest driver of stress in the CST cohort, career development, which from the statements collected, resulted from the heat and burden of acquiring the academic evidence necessary to support higher surgical NTN appointment. To date, only one cohort has completed the IST pilot, but if early results are associated with less burnout, this would support a drive towards complete CST reconfiguration with IST strategic rotations.

This study has a number of limitations. Questionnaire methodology has inherent disadvantages, primarily that two-way communication cannot be achieved, to clarify the meaning underlying a respondent's answer, and viceversa, respondents may struggle to gain clarity regarding the focus of questions asked. Moreover, some questions risk eliciting no response. In contrast, the study has strengths. The response rate was good and the number of statements generated were high, with common themes emerging. The questionnaires were completed voluntarily and anonymously in a holding room prior to the trainees' ARCPs, resulting in minimal intertrainee communication regarding answers, because only two to three were present at any given time, more likely resulting in genuine personal views. Finally, inter-rater reliability and agreement ranged from good to excellent for all statements reviewed. 
In conclusion, Michie's model has again been shown to be an effective means to analyse and report stress and burn-out causality among doctors, on this occasion CSTs. The information gathered from this study illustrates the need for pressing change in the structure of UK CST, allied to an enhanced training environment in general, to protect the future surgical workforce and ensure an equitable training experience for all. Doctors regardless of gender or training grade deserve a safe, supportive environment in which to work and develop.

Twitter David Bryan Thomas Robinson @daverobinson90, Luke Hopkins @luke_ hopkins, Arfon GMT Powell @PowArG07 and Rhiannon L Harries @rhiharries

Contributors DBTR: concept and design, data acquisition and analysis, drafting, final approval and submission. OL: data analysis, drafting, final approval. KM; data acquisition, critical revision and final approval. OPJ: data acquisition, drafting and final approval. LH: design, data acquisition, drafting and final approval. AP: data interpretation, critical revision and final approval. SH-G: concept, data acquisition, critical revision and final approval. RLH: design, drafting, critical revision and final approval. RE: concept, data acquisition, critical revision and final approval. WGL: concept, drafting, final approval prior to submission, content guarantor.

Funding OPJ is supported by a Joint Surgical Research Fellowship from the Royal College of Surgeons of England and Health Education and Improvement Wales. Award/Grant number is not applicable.

Competing interests None declared.

Patient consent for publication Not required.

Ethics approval This study represents evaluation of the challenges faced within the core surgical training programme. Completion of questionnaires was voluntary and anonymous and therefore ethical approval was not required.

Provenance and peer review Not commissioned; externally peer reviewed.

Data availability statement All data relevant to the study are included in the article or uploaded as online supplemental information. A table outlining categorisation of the statements provided can be made available by contacting the corresponding author.

Supplemental material This content has been supplied by the author(s). It has not been vetted by BMJ Publishing Group Limited (BMJ) and may not have been peer-reviewed. Any opinions or recommendations discussed are solely those of the author(s) and are not endorsed by BMJ. BMJ disclaims all liability and responsibility arising from any reliance placed on the content. Where the content includes any translated material, BMJ does not warrant the accuracy and reliability of the translations (including but not limited to local regulations, clinical guidelines, terminology, drug names and drug dosages), and is not responsible for any error and/or omissions arising from translation and adaptation or otherwise.

Open access This is an open access article distributed in accordance with the Creative Commons Attribution Non Commercial (CC BY-NC 4.0) license, which permits others to distribute, remix, adapt, build upon this work non-commercially, and license their derivative works on different terms, provided the original work is properly cited, appropriate credit is given, any changes made indicated, and the use is non-commercial. See: http://creativecommons.org/licenses/by-nc/4.0/.

\section{ORCID iDs}

David Bryan Thomas Robinson http://orcid.org/0000-0003-1087-744X

Oliver Luton http://orcid.org/0000-0001-9049-6879

Katie Mellor http://orcid.org/0000-0002-3556-5701

Osian Penri James http://orcid.org/0000-0002-1986-1391

Luke Hopkins http://orcid.org/0000-0003-1218-9907

Arfon GMT Powell http://orcid.org/0000-0002-3740-8275

\section{REFERENCES}

1 Luo $\mathrm{H}$, Yang $\mathrm{H}, \mathrm{Xu} \mathrm{X}$, et al. Relationship between occupational stress and job burnout among rural-to-urban migrant workers in Dongguan, China: a cross-sectional study. BMJ Open 2016;6:e012597.

2 Maslach C, Jackson SE. The measurement of experienced burnout. J Organ Behav 1981;2:99-113.

3 World Health Organisation. Burn-out an 'occupational phenomenon': International Classification of Diseases, 2019

4 Balch CM. Stress and burnout among surgeons. Arch Surg 2009;144:371-6.

5 Michie S. Causes and management of stress at work. Occup Environ Med 2002;59:67-72.

6 Murphy LR. Occupational stress management: current status and future directions. Trends Organ Behav 1995;2:1-14.

7 Tallentire VR, Smith SE, Facey AD, et al. Exploring newly qualified doctors' workplace stressors: an interview study from Australia. BMJ Open 2017;7:e015890.

8 Robinson DBT, James OP, Hopkins L, et al. Stress and burnout in training; requiem for the surgical DREAM. J Surg Educ 2020;77:e1-8.

9 McHugh ML. Interrater reliability: the kappa statistic. Biochem Med 2012;22:276-82.

10 Altman D. Practical statistics for medical research. Chapman and. 1991. London: Hall, 1991.

11 Bogg J, Gibbs T, Bundred P. Training, job demands and mental health of pre-registration house officers. Med Educ 2001;35:590-5.

12 Walker NR, Deekonda P, Glasbey JC, et al. Attracting medical students and doctors into surgical training in the UK and Ireland. Int J Surg 2019;67:107-12.

13 General Medical Council. The state of medical education and practice in the UK, 2017. Available: https://www.gmc-uk.org/static/ documents/content/SoMEP-2017-final-full.pdf

14 Hunt V, Layton D, Prince S. Why diversity matters: Diversity's dividend, 2015. Available: https://www.mckinsey.com/businessfunctions/organization/our-insights/why-diversity-matters

15 Shannon G, Jansen M, Williams K, et al. Gender equality in science, medicine, and global health: where are we at and why does it matter? Lancet 2019;393:560-9.

16 Wallis CJ, Ravi B, Coburn N, et al. Comparison of postoperative outcomes among patients treated by male and female surgeons: a population based matched cohort study. BMJ 2017;359:j4366. 